\title{
A multidomain spectral method for solving elliptic equations
}

\author{
Harald P. Pfeiffer团, Lawrence E. Kidder H, Mark A. Schee用, and Saul A. \\ Teukolsky \\ * Department of Physics, Cornell University, Ithaca, New York 14853, † Center \\ for Radiophysics and Space Research, Cornell University, Ithaca, New York \\ 14853, $\ddagger$ California Institute of Technology, Pasadena, California 91125, and \\ $\S$ Department of Astrophysics, American Museum of Natural History, CPW $\&$ \\ 79th Street, New York, NY 10024. Permanent address: Department of Physics, \\ Cornell University \\ E-mail: harald@astro.cornell.edu,kidder@astro.cornell.edu, \\ scheel@tapir.caltech.edu, saul@astro.cornell.edu
}

Version: February 27, 2002

We present a new solver for coupled nonlinear elliptic partial differential equations (PDEs). The solver is based on pseudo-spectral collocation with domain decomposition and can handle one- to three-dimensional problems. It has three distinct features. First, the combined problem of solving the PDE, satisfying the boundary conditions, and matching between different subdomains is cast into one set of equations readily accessible to standard linear and nonlinear solvers. Second, touching as well as overlapping subdomains are supported; both rectangular blocks with Chebyshev basis functions as well as spherical shells with an expansion in spherical harmonics are implemented. Third, the code is very flexible: The domain decomposition as well as the distribution of collocation points in each domain can be chosen at run time, and the solver is easily adaptable to new PDEs. The code has been used to solve the equations of the initial value problem of general relativity and should be useful in many other problems. We compare the new method to finite difference codes and find it superior in both runtime and accuracy, at least for the smooth problems considered here.

Key Words: spectral methods; domain decomposition; general relativity

\section{INTRODUCTION}

Elliptic partial differential equations (PDE) are a basic and important aspect of almost all areas of natural science. Numerical solutions of PDEs in three or more dimensions pose a formidable problem, requiring a significant amount of memory and CPU time. Off-the-shelf solvers are available; however, it can be difficult to adapt such a solver to a particular problem at hand, especially when the computational domain of the PDE is nontrivial, or when one deals with a set of coupled PDEs.

There are three basic approaches to solving PDEs: Finite differences, finite elements and spectral methods. Finite differences are easiest to code. However, they converge only algebraically and therefore need a large number of grid points and have correspondingly large memory requirements. Finite elements and spectral methods both expand the solution in basis functions. Finite elements use many 
subdomains and expand to low order in each subdomain, whereas spectral methods use comparatively few subdomains with high expansion orders. Finite elements are particularly well suited to irregular geometries appearing in many engineering applications. For sufficiently regular domains, however, spectral methods are generally faster and/or more accurate.

Multidomain spectral methods date back at least to the work of Orszag 22]. In a multidomain spectral method, one has to match the solution across different subdomains. Often this is accomplished by a combination of solves on individual subdomains together with a global scheme to find the function values at the internal subdomain boundaries. Examples of such global schemes are relaxational iteration 13], an influence matrix 19, [], or the spectral projection decomposition method 14]. For simple PDEs like the Helmholtz equation, fast solvers for the subdomain solves are available. For more complicated PDEs, or for coupled PDEs, the subdomain solves will typically use an iterative solver. One drawback of these schemes is that information from the iterative subdomain solves is not used in the global matching procedure until the subdomain solves have completely converged. The question arises whether efficiency can be improved by avoiding this delay in communication with the matching procedure.

In this paper we present a new spectral method for coupled nonlinear PDEs based on pseudospectral collocation with domain decomposition. This method does not split subdomain solves and matching into two distinct elements. Instead it combines satisfying the PDE on each subdomain, matching between subdomains, and satisfying the boundary conditions into one set of equations. This system of equations is then solved with an iterative solver, typically GMRES[5]. At each iteration, this solver thus has up-to-date information about residuals on the individual subdomains and about matching and thus can make optimal use of all information.

The individual subdomains implemented are rectangular blocks and spherical shells. Whereas either rectangular blocks (see e.g. [11, 18, 24]) or spherical shells 16 have been employed before, we are not aware of work using both. The code supports an arbitrary number of blocks and shells that can touch each other and/or overlap.

Moreover, the operator $\mathcal{S}$ at the core of the method (see section 3.3) turns out to be modular, i.e. the code fragments used to evaluate the PDE, the boundary conditions, and the matching conditions are independent of each other. Thus the structure of the resulting code allows for great flexibility, which is further enhanced by a novel point of view of the mappings that are used to map collocation coordinates to the physical coordinates. This flexibility is highlighted in the following aspects:

- The user code for the particular PDE at hand is completely independent from the code dealing with the spectral method and domain decomposition. For a new PDE, the user has to supply only the code that computes the residual and its linearization.

- Mappings are employed to control how collocation points and thus resolution are distributed within each subdomain. New mappings can be easily added which are then available for all PDEs that have been coded.

- The solver uses standard software packages for the Newton-Raphson step, the iterative linear solvers, and the preconditioning. Thus one can experiment with many different linear solvers and different preconditioners to find 
an efficient combination. The code will also automatically benefit from improvements to these software packages.

- The code is dimension independent (up to three dimensions).

- Many properties of a particular solve can be chosen at runtime, for example the domain decomposition, the mappings used in each subdomain, as well as the choice of the iterative solver. The user can also choose among differential operators and boundary conditions previously coded at runtime.

In the next section we recall some basics of the pseudo-spectral collocation method. In section 3 we describe our novel approach of combining matching with solving of the PDE. For ease of exposition, we interweave the new method with more practical issues like mappings and code modularity. The central piece of our method, the operator $\mathcal{S}$, is introduced in section 3.3 for a one-dimensional problem and then extended to higher dimensions and spherical shells. In section 16 we solve three example problems. Many practical issues like preconditioning and parallelization are discussed in this section, and we also include a detailed comparison to a finite difference code.

\section{SPECTRAL METHODS}

We deal with a second order nonlinear elliptic partial differential equation or system of equations,

$$
(\mathcal{N} u)(x)=0, \quad x \in \mathcal{D},
$$

in some domain $\mathcal{D} \subset \mathbb{R}^{d}$ with boundary conditions

$$
g(u)(x)=0, \quad x \in \partial \mathcal{D} .
$$

The function $u$ can be a single variable giving rise to a single PDE, or it can be vector-valued giving rise to a coupled set of PDEs. Throughout this paper we assume that the problem has a unique solution. We also absorb a possible righthand side into the elliptic operator $\mathcal{N}$.

The fundamental idea of spectral methods is to approximate the solution to the $\operatorname{PDE}(1)$ as a series in some basis functions $\Phi_{k}(x)$ :

$$
u(x) \approx u^{(N)}(x) \equiv \sum_{k=0}^{N} \tilde{u}_{k} \Phi_{k}(x) .
$$

The coefficients $\tilde{u}_{k}$ are called the spectral coefficients. The power of spectral methods stems from two simple facts:

1. When approximating a smooth function with a series (3), the error of the approximation decreases exponentially with the number of basis functions $N$. Hence $u^{(N)}$ will converge toward the true solution $u$ of the PDE exponentially, provided $u$ is smooth and one can determine the spectral coefficients $\tilde{u}_{k}$ sufficiently well.

2. One can evaluate the action of $\mathcal{N}$ on the function $u^{(N)}$ exactly (i.e. up to numerical round-off). This fact allows one to find the $\tilde{u}_{k}$ accurately. 
The second fact arises because the derivatives of the basis functions are known analytically, and so by Eq. (3),

$$
\frac{d u^{(N)}(x)}{d x}=\sum_{k=0}^{N} \tilde{u}_{k} \frac{d \Phi_{k}(x)}{d x},
$$

and similarly for higher derivatives.

In order to compute the spectral coefficients $\tilde{u}_{k}$ we use pseudo-spectral collocation where one requires

$$
\left(\mathcal{N} u^{(N)}\right)\left(x_{i}\right)=0, \quad i=0, \ldots, N .
$$

The points $x_{i}$ are called collocation points, and are chosen as the abscissas of the Gaussian quadrature associated with the basis set $\Phi_{k}$. This choice of collocation points can be motivated by considering $\int\left[\left(\mathcal{N} u^{(N)}\right)(x)\right]^{2} d x$. Evaluating this integral with Gaussian quadrature, we find by virtue of Eq. (5)

$$
\int_{\mathcal{D}}\left[\left(\mathcal{N} u^{(N)}\right)(x)\right]^{2} d x \approx \sum_{i=0}^{N} w_{i}\left[\left(\mathcal{N} u^{(N)}\right)\left(x_{i}\right)\right]^{2}=0
$$

where $w_{i}$ are the weights of the quadrature. We see that $\mathcal{N} u^{(N)}$ must be small throughout $\mathcal{D}$ and thus the function $u^{(N)}$ satisfying Eqs. (5) must be close to the true solution. More rigorous treatments of the pseudospectral collocation method can be found in the literature $15,8,6$.

\subsection{Chebyshev polynomials}

Chebyshev polynomials are widely used as basis functions for spectral methods. They satisfy the convenient analytical properties of "classical" orthogonal polynomials. Their defining differential equation is a singular Sturm-Liouville problem, and so Chebyshev expansions converge exponentially for smooth functions $u$ independent of the boundary conditions satisfied by $u[15,6$.

Chebyshev polynomials are defined by

$$
T_{k}(X)=\cos (k \arccos X), \quad X \in[-1,1] .
$$

They are defined on the interval $X \in[-1,1]$ only; usually one needs to map the collocation coordinate $X \in[-1,1]$ to the physical coordinate of the problem, $x \in$ $[a, b]$. We use the convention that the variable $X$ varies over the interval $[-1,1]$, whereas $x$ is defined over arbitrary intervals. We will describe our approach to mappings below in the implementation section.

For an expansion up to order $N$ (i.e. having a total of $N+1$ basis functions) the associated collocation points are

$$
X_{i}=\cos \left(\frac{i \pi}{N}\right), \quad i=0, \ldots, N .
$$

Define the real space values

$$
u_{i} \equiv u^{(N)}\left(X_{i}\right)=\sum_{k=0}^{N} \tilde{u}_{k} T_{k}\left(X_{i}\right) .
$$


Using the discrete orthogonality relation

$$
\delta_{j k}=\frac{2}{N \bar{c}_{k}} \sum_{i=0}^{N} \frac{1}{\bar{c}_{i}} T_{j}\left(X_{i}\right) T_{k}\left(X_{i}\right)
$$

with

$$
\bar{c}_{i}= \begin{cases}2 & k=0 \text { or } k=N \\ 1 & k=1, \ldots, N-1,\end{cases}
$$

we can invert (9) and find

$$
\tilde{u}_{j}=\frac{2}{N \bar{c}_{j}} \sum_{i=0}^{N} \frac{u_{i}}{\bar{c}_{i}} T_{j}\left(X_{i}\right) .
$$

Both matrix multiplications (9) and (12) can be performed with a fast cosine transform in $\mathcal{O}(N \ln N)$ operations, another reason for the popularity of Chebyshev basis functions.

There are the same number of real space values $u_{i}$ and spectral coefficients $\tilde{u}_{k}$, and there is a one-to-one mapping between $\left\{u_{i}\right\}$ and $\left\{\tilde{u}_{k}\right\}$. Hence one can represent the function $u^{(N)}$ by either $\left\{u_{i}\right\}$ or $\left\{\tilde{u}_{k}\right\}$.

The spectral coefficients of the derivative,

$$
\frac{d u^{(N)}}{d X}(X)=\sum_{k=0}^{N} \tilde{u}_{k}^{\prime} T_{k}(X),
$$

are given by the recurrence relation

$$
\begin{aligned}
& \tilde{u}_{i}^{\prime}=\tilde{u}_{i+2}^{\prime}+2(i+1) \tilde{u}_{i+1}, \quad i=1, \ldots, N-1, \\
& \tilde{u}_{0}^{\prime}=\frac{1}{2} \tilde{u}_{2}^{\prime}+\tilde{u}_{1},
\end{aligned}
$$

with $\tilde{u}_{N+1}=\tilde{u}_{N}=0$. The coefficients of the second derivative,

$$
\frac{d^{2} u^{(N)}}{d X^{2}}(X)=\sum_{k=0}^{N-1} \tilde{u}_{k}^{\prime \prime} T_{k}(X),
$$

are obtained by a similar recurrence relation, or by applying (14) twice.

\subsection{Basis functions in higher dimensions}

In higher dimensions one can choose tensor grids of lower dimensional basis functions. For example, a $d$-dimensional cube $[-1,1]^{d}$ can be described by Chebyshev polynomials along each coordinate axis. For a three-dimensional sphere or spherical shell, tensor products of spherical harmonics for the angles and a Chebyshev series for the radial coordinate 16 are used. It is also possible to expand the angular piece in a double Fourier-series 21 . 


\subsection{Domain Decomposition}

If the computational domain $\mathcal{D}$ has a different topology than the basis functions, then an expansion in the basis functions cannot cover $\mathcal{D}$ completely. Moreover, the particular problem at hand might require different resolution in different regions of the computational domain which will render a single overall expansion inefficient.

One circumvents these problems with domain decomposition. The computational domain $\mathcal{D}$ is covered by $N_{\mathcal{D}}$ subdomains

$$
\mathcal{D}=\bigcup_{\mu=1}^{N_{\mathcal{D}}} \mathcal{D}_{\mu}
$$

each having its own set of basis functions and expansion coefficients:

$$
u^{(\mu)}(x)=\sum_{k=0}^{N_{\mu}} \tilde{u}_{k}^{(\mu)} \Phi_{k}^{(\mu)}(x), \quad x \in \mathcal{D}_{\mu}, \quad \mu=1, \ldots N_{\mathcal{D}} .
$$

Here $u^{(\mu)}$ denotes the approximation in the $\mu$-th domain, and we have dropped the additional label $N$ denoting the expansion order of $u^{(\mu)}$. The individual subdomains $\mathcal{D}_{\mu}$ can touch each other or overlap each other. To ensure that the functions $u^{(\mu)}$ - each defined on a single subdomain $\mathcal{D}_{\mu}$ only - actually fit together and form a smooth solution of the PDE (1) on the full domain $\mathcal{D}$, they have to satisfy matching conditions. In the limit of infinite resolution, we must have that

- for touching subdomains $\mathcal{D}_{\mu}$ and $\mathcal{D}_{\nu}$ the function and its normal derivative must be smooth on the surface where the subdomains touch:

$$
\begin{aligned}
u^{(\mu)}(x) & =u^{(\nu)}(x) \\
\frac{\partial u^{(\mu)}}{\partial n}(x) & =-\frac{\partial u^{(\nu)}}{\partial n}(x) \quad x \in \partial \mathcal{D}_{\mu} \cap \partial \mathcal{D}_{\nu}
\end{aligned}
$$

(The minus sign in the second equation of (18) occurs because we use the outward-pointing normal in each subdomain.)

- for overlapping subdomains $\mathcal{D}_{\mu}$ and $\mathcal{D}_{\nu}$ the functions $u^{(\mu)}$ and $u^{(\nu)}$ must be identical in $\mathcal{D}_{\mu} \cap \mathcal{D}_{\nu}$. By uniqueness of the solution of the PDE, it suffices to require that the functions are identical on the boundary of the overlapping domain:

$$
u^{(\mu)}(x)=u^{(\nu)}(x), \quad x \in \partial\left(\mathcal{D}_{\mu} \cap \mathcal{D}_{\nu}\right) .
$$

We will see in the next section how these conditions are actually implemented in the code.

\section{IMPLEMENTATION}

In this section we describe our specific approaches to several aspects of multidimensional pseudo-spectral collocation with domain decomposition. 


\subsection{One-dimensional Mappings}

Chebyshev polynomials are defined for $X \in[-1,1]$. Differential equations in general will be defined on a different interval $x \in[a, b]$. In order to use Chebyshev polynomials, one introduces a mapping

$$
X:[a, b] \rightarrow[-1,1], \quad x \rightarrow X=X(x)
$$

that maps the physical coordinate $x$ onto the collocation coordinate $X$.

One could explicitly substitute this mapping into the PDE under consideration. Derivatives would be multiplied by a Jacobian, and we would obtain the PDE on the interval $[-1,1]$. For example, the differential equation in the variable $x$

$$
\frac{\partial^{2} u}{\partial x^{2}}+u=0, \quad x \in[a, b]
$$

becomes the following differential equation in the variable $X$ :

$$
X^{\prime 2} \frac{\partial^{2} u}{\partial X^{2}}+X^{\prime \prime} \frac{\partial u}{\partial X}+u=0, \quad X \in[-1,1],
$$

where $X^{\prime}=\partial X / \partial x$ and $X^{\prime \prime}=\partial^{2} X / \partial x^{2}$. Now one could expand $u(X)$ in Chebyshev polynomials, compute derivatives $\partial / \partial X$ via the recurrence relation (14) and code Eq. (22) in terms of $\partial u / \partial X$. This approach is common in the literature [6, 17]. However, it has several disadvantages: As one can already see from this simple example, the equations become longer and one has to code and debug more terms. Second, and more important, it is inflexible, since for each different map one has to derive and code a mapped equation (22). A priori one might not know the appropriate map for a differential equation, and in order to try several maps, one has to code the mapped equation several times. Also, for domain decomposition, a different map is needed for each subdomain.

We propose a different approach. We still expand in terms of Chebyshev polynomials on $X \in[-1,1]$ and obtain the physical solution via a mapping $X(x)$,

$$
u(x)=\sum_{k=0}^{N} \tilde{u}_{k} T_{k}(X(x)),
$$

and we still compute $\partial u(X) / \partial X$ and $\partial^{2} u(X) / \partial X^{2}$ via the recurrence relation (14). However, now we do not substitute $\partial u(X) / \partial X$ and $\partial^{2} u(X) / \partial X^{2}$ into the mapped differential equation, Eq. (22). Instead we compute first numerically

$$
\begin{aligned}
\frac{\partial u(x)}{\partial x} & =X^{\prime} \frac{\partial u(X)}{\partial X} \\
\frac{\partial^{2} u(x)}{\partial x^{2}} & =X^{\prime 2} \frac{\partial^{2} u(X)}{\partial X^{2}}+X^{\prime \prime} \frac{\partial u(X)}{\partial X}
\end{aligned}
$$

and substitute these values into the original physical differential equation (21). The collocation points are thus mapped to the physical coordinates

$$
x_{i}=X^{-1}\left(X_{i}\right) .
$$

This approach separates the code into three distinct parts: 
1. Code dealing with the basis functions: transforms between collocation space $X$ and spectral space, evaluation of derivatives $\partial / \partial X$ via recurrence relations. This code depends only on the collocation coordinates $X \in[-1,1]$ (and on the angular coordinates $\theta, \phi$ for spherical shells).

2. Mappings that map between collocation coordinate $X$ and physical coordinates $x$.

3. The "user code" implementing the physical PDE [in our example Eq. (21)] that deals only with the physical coordinate $x$.

These three elements are independent of each other:

- A user who wants to code another differential equation has only to write the code that evaluates the differential operator $\mathcal{N}$ in the physical space with physical derivatives. Then immediately all previously coded mappings are available for this new differential equation, as well as all basis functions.

- In order to introduce a new mapping, one has to code only four functions, namely $X(x)$, its inverse $x(X)$, as well as the derivatives $X^{\prime}(x)$ and $X^{\prime \prime}(x)$. This new map can then be used for any differential equation already coded or to be coded later.

- In order to switch to a different set of basis functions, one has only to code the transforms and the recurrence relations for the derivatives.

In practice we use three different mappings

$$
\begin{array}{ll}
\text { linear: } & X(x)=A x+B \\
\text { log: } & X(x)=A \log (B x+C) \\
\text { inverse: } & X(x)=\frac{A}{x}+B
\end{array}
$$

In each case the constants $A, B$ are chosen such that $[a, b]$ is mapped to $[-1,1]$. The log mapping has one additional parameter which is used to fine-tune the relative density of collocation points at both ends of the interval $[a, b]$. We show the effects of different mappings in our first example in section 4.1.

\subsection{Basis functions and Mappings in higher Dimensions}

\subsubsection{Rectangular Blocks}

In order to expand in a $d$-dimensional rectangular block,

$$
\mathcal{D}=\left[a_{1}, b_{1}\right] \times\left[a_{2}, b_{2}\right] \times \ldots \times\left[a_{d}, b_{d}\right],
$$

we use a tensor product of Chebyshev polynomials with a 1- $d$ mapping along each coordinate axis:

$$
u\left(x_{1}, \ldots, x_{d}\right)=\sum_{k_{1}=0}^{N_{1}} \sum_{k_{2}=0}^{N_{2}} \cdots \sum_{k_{d}=0}^{N_{d}} \tilde{u}_{k_{1} \cdots k_{d}} T_{k_{1}}\left(X^{(1)}\left(x_{1}\right)\right) \cdots T_{k_{d}}\left(X^{(d)}\left(x_{d}\right)\right) .
$$

We use $d$ mappings

$$
X^{(l)}:\left[a_{l}, b_{l}\right] \rightarrow[-1,1], \quad l=1, \ldots d,
$$


and the collocation points in physical space are the mapped collocation points along each dimension,

$$
x_{i_{1} \ldots i_{d}}=\left(x_{i_{1}}^{(1)}, \ldots, x_{i_{d}}^{(d)}\right)
$$

where the coordinate along the $l$-th dimension $x_{i_{l}}^{(l)}$ is given by Eq. (26) using $X^{(l)}$.

Note that such a $d$-dimensional rectangle has as many spectral coefficients $\tilde{u}_{k_{1} \cdots k_{d}}$ as grid point values $u_{i_{1} \ldots i_{d}}=u\left(x_{i_{1}}, \ldots, x_{i_{d}}\right)$. Therefore we can equivalently solve for the spectral coefficients or the real space values. We will solve for the real space values $u_{i_{1} \ldots i_{d}}$.

\subsubsection{Spherical Shell}

In a spherical shell with inner and outer radii $0<R_{1}<R_{2}$ we use a mapping for the radial coordinate. A function $u(r, \theta, \phi)$ is thus expanded as

$$
u(r, \theta, \phi)=\sum_{k=0}^{N_{r}} \sum_{l=0}^{L} \sum_{m=-l}^{l} \tilde{u}_{k l m} T_{k}(X(r)) Y_{l m}(\theta, \phi),
$$

where real-valued spherical harmonics are used:

$$
Y_{l m}(\theta, \phi) \equiv \begin{cases}P_{l}^{m}(\cos \theta) \cos (m \phi), & m \geq 0 \\ P_{l}^{|m|}(\cos \theta) \sin (|m| \phi), & m<0\end{cases}
$$

$P_{l}^{m}(\cos \theta)$ are the associated Legendre polynomials. Associating the sin-terms with negative $m$ is not standard, but eliminates the need to refer to two sets of spectral coefficients, one each for the cos-terms and the sin-terms. The radial mapping $X:\left[R_{1}, R_{2}\right] \rightarrow[-1,1]$ can be any of the choices in Eq. (27). The radial collocation points $r_{i}, i=0, \ldots, N_{r}$ are given by Eq. (26).

For the angle $\phi$, Eq. (33) leads to a Fourier series with equally spaced azimuthal collocation points

$$
\phi_{i}=\frac{2 \pi i}{N_{\phi}}, \quad i=0,1, \ldots, N_{\phi}-1 .
$$

There is a total of $N_{\theta}=L+1$ angular collocation points $\theta_{i}$, which are the abscissas of Gauss-Legendre integration.

We employ the software package Spherepack [1] which provides routines to compute the collocation points, transforms and angular derivatives.

In order to resolve the full Fourier series in $\phi$ up to $m=L$, one needs $N_{\phi} \geq$ $2 L+1$, since for $N_{\phi}=2 L$, the term $\sin (L \phi)$ vanishes at all collocation points $\phi_{i}$. We use $N_{\phi}=2(L+1)$ since FFTs are more efficient with an even number of points.

The expansion (32) has a total of $\left(N_{r}+1\right)(L+1)^{2}$ spectral coefficients but a total of $\left(N_{r}+1\right) N_{\theta} N_{\phi}=2\left(N_{r}+1\right)(L+1)^{2}$ collocation points. This means a spherical shell has more collocation points than spectral coefficients and the expansion (32) approximates the grid point values in a least-square sense only 28]. Performing a spectral transform and its inverse will thus project the grid point values into a subspace with dimension equal to the number of spectral coefficients. The implications of this fact for our code are discussed below in section 3.6. 


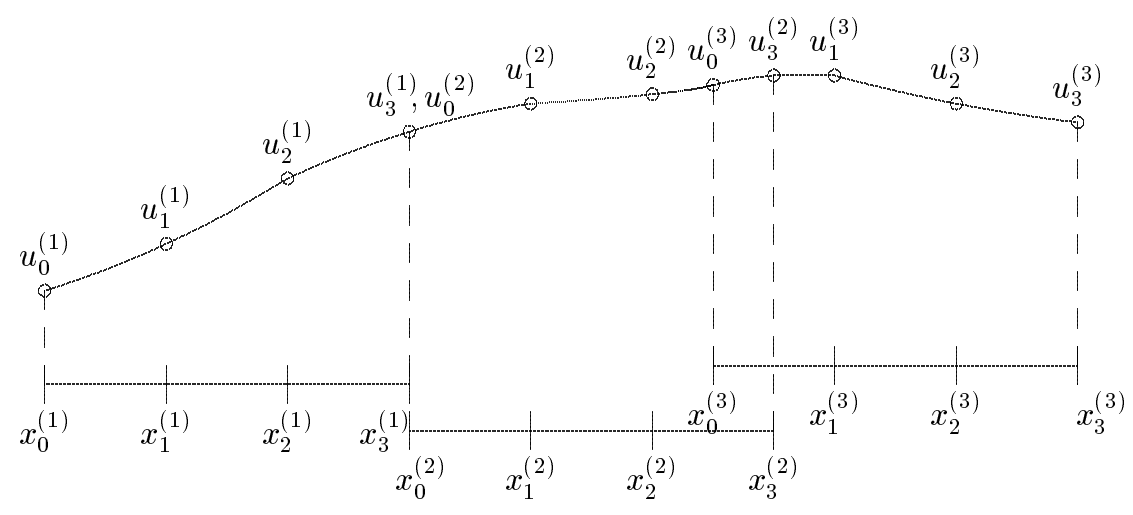

Domain 1

Domain 2

Domain 3

FIG. 1 Illustration of matching with three subdomains in one dimension. Subdomains 1 and 2 touch each other, and subdomains 2 and 3 overlap. $x_{i}^{(\mu)}$ denotes the coordinate of the $i$-th collocation point of the $\mu$-th subdomain, and $u_{i}^{(\mu)}$ denotes the function values at the grid points.

\subsubsection{Representation of vectors and derivatives}

In both kinds of subdomains, rectangular blocks and spherical shells, we expand the Cartesian components of vectors, and we compute Cartesian derivatives, $\partial / \partial x, \partial / \partial y, \partial / \partial z$. These quantities are smooth everywhere, and thus can be expanded in scalar spherical harmonics. By contrast, the spherical components of a vector field in a spherical shell are discontinuous at the poles and cannnot be expanded in scalar spherical harmonics. One would have to use e.g. vector spherical harmonics 28, 29.

For a spherical shell we provide an additional wrapper around the basis functions and the radial mapping that transforms polar derivatives $\partial / \partial r, \partial / \partial \theta, \partial / \partial \phi$ to Cartesian derivatives. This involves multiplications by sines and cosines of the angles $\theta, \phi$ which can be performed grid point by grid point in real space. Alternatively, it can be done spectrally by expressing, e.g. $\sin \theta$ in spherical harmonics, and then using addition theorems to reduce products of spherical harmonics to simple sums. Carrying out the transformation spectrally is slightly better in practice.

Representing vectors and derivatives in Cartesian coordinates in both kinds of subdomains increases flexibility, too. We can use the same code to evaluate the residual in both kinds of subdomains.

\subsection{The operator $\mathcal{S}$}

We now introduce the operator $\mathcal{S}$, the centerpiece of our method. It combines the solution of the PDE, the boundary conditions and matching between different subdomains.

We introduce $\mathcal{S}$ first with a simple case, a one-dimensional differential equation with a Dirichlet boundary condition at one end and a von Neumann boundary condition at the other end: 


$$
\begin{aligned}
(\mathcal{N} u)(x) & =0, \quad a<x<b, \\
u(a) & =A, \\
\frac{\partial u}{\partial x}(b) & =B .
\end{aligned}
$$

To explain our procedure for matching, we assume three domains as depicted in Figure 1. $N_{\mu}$ denotes the highest retained expansion order in domain $\mu$; here $N_{\mu}=3$ for all domains. Domains 2 and 3 overlap. Domains 1 and 2 touch so that the collocation points $x_{3}^{(1)}$ and $x_{0}^{(2)}$ represent the same physical point. The function value, however, is represented twice, once assigned to domain $1, u_{3}^{(1)}$, and once belonging to domain $2: u_{0}^{(2)}$. Using just the grid point values within one subdomain, we can expand the function in that subdomain and can evaluate derivatives. We can also interpolate the function to arbitrary positions $x$. Thus, given values $\left\{u_{i}^{(\mu)}, i=0, \ldots, N_{\mu}\right\}$, we can compute $u^{(\mu)}(x)$ for $x \in\left[x_{0}^{(\mu)}, x_{N_{\mu}}^{(\mu)}\right]$.

In order to determine the unknowns $u_{i}^{(\mu)}$, we need one equation per unknown. We will first write down these equations and then explain where they come from.

$$
\begin{aligned}
\left(\mathcal{N} u^{(\mu)}\right)\left(x_{i}^{(\mu)}\right) & =0, \quad i=1, \ldots, N_{\mu}-1, \quad \mu=1, \ldots, N_{\mathcal{D}} \\
u_{0}^{(1)}-A & =0 \\
\frac{\partial u^{(3)}}{\partial x}\left(x_{3}^{(3)}\right)-B & =0 \\
u_{3}^{(1)}-u_{0}^{(2)} & =0 \\
\frac{\partial u^{(1)}}{\partial n}\left(x_{3}^{(1)}\right)+\frac{\partial u^{(2)}}{\partial n}\left(x_{3}^{(1)}\right) & =0 \\
u_{3}^{(2)}-u^{(3)}\left(x_{3}^{(2)}\right) & =0 \\
u_{0}^{(3)}-u^{(2)}\left(x_{0}^{(3)}\right) & =0
\end{aligned}
$$

Eq. (38a) represents the actual pseudo-spectral equation (5). It is enforced only for collocation points that are not on the boundary of a subdomain. Eqs. (38b) and (38d) encode the boundary conditions. Eqs. (38d) and 38d describe the value and derivative matching at touching subdomain boundaries. These equations follow from Eq. (18). Eqs. (381) and (38g) perform matching between overlapping subdomains as given by Eq. (19).

We will view the left-hand side of Eqs. (38) as a non-linear operator $\mathcal{S}$. This operator acts on the set of grid point values for all subdomains $\left\{u_{i}^{(\mu)}\right\}(\mu=$ $1,2,3, i=0, \ldots, N_{\mu}$ in the example) and returns a residual that incorporates the actual pseudo-spectral condition Eq. (5), the boundary conditions, and the matching conditions between different subdomains. If we denote the vector of all grid point values by $\underline{\mathbf{u}}$, then the discretized version of the partial differential equation becomes

$$
\mathcal{S} \underline{\mathbf{u}}=0 \text {. }
$$

The solution of Eq. (39) clearly is the solution of the partial differential equation we want to obtain. By virtue of Eq. (39) we thus have condensed the PDE, the boundary conditions and matching into one set of nonlinear equations.

We comment on some implementation issues: 
- The action of the operator $\mathcal{S}$ can be computed very easily: Given grid point values $\underline{\mathbf{u}}$, every subdomain is transformed to spectral space and derivatives are computed. Using the derivatives we can compute Eqs. (38a), (38d) and any boundary conditions that involve derivatives like Eq. (38d). The interpolations necessary in Eqs. (38f) and (38g) are done by summing up the spectral series.

- $\mathcal{S} \underline{\mathbf{u}}$ can be computed in parallel: Everything except the matching conditions depends only on the set of grid point values within one subdomain. Therefore the natural parallelization is to distribute subdomains to different processors.

- The code fragments implementing the nonlinear operator $\mathcal{N}$, the boundary conditions and the matching conditions are independent of each other. In order to change boundary conditions, one has only to modify the code implementing Eqs. (38b) and (38c). In particular, the code for the matchingequations (38d)-(38g) can be used for any differential operator $\mathcal{N}$ and for any boundary condition.

We have now introduced the operator $\mathcal{S}$ in one dimension. Next we address how to solve Eq. (39), and then we generalize $\mathcal{S}$ to higher dimensions. We present our method in this order because the generalization to higher dimensions depends on some details of the solution process.

\subsection{Solving $\mathcal{S} u=0$}

In this section we describe how we solve the system of nonlinear equations (39). Our procedure is completely standard and requires three ingredients: A NewtonRaphson iteration to reduce the nonlinear equations to a linear solve at each iteration, an iterative linear solver, and the preconditioner for the linear solver. For these three steps we employ the software package PETSc 伍. We now comment on each of these three stages.

\subsubsection{Newton-Raphson with line searches}

PETSc [1 implements a Newton-Raphson method with line searches, similar to the method described in 25]. Given a current guess $\underline{\mathbf{u}}_{\text {old }}$ of the solution, a NewtonRaphson step proceeds as follows: Compute the residual

$$
\underline{\mathbf{r}} \equiv \mathcal{S} \underline{\mathbf{u}}_{\text {old }}
$$

and linearize $\mathcal{S}$ around the current guess $\underline{\mathbf{u}}_{\text {old }}$ of the solution:

$$
\mathcal{J} \equiv \frac{\partial \mathcal{S}}{\partial \underline{\mathbf{u}}}\left(\underline{\mathbf{u}}_{\text {old }}\right) .
$$

The Jacobian $\mathcal{J}$ is a $N_{D F} \times N_{D F}$-dimensional matrix, $N_{D F}$ being the number of degrees of freedom. Next compute a correction $\delta \underline{\mathbf{u}}$ by solving the linear system

$$
\mathcal{J} \delta \underline{\mathbf{u}}=-\underline{\mathbf{r}} .
$$

Finally a line-search is performed in the direction of $\delta \underline{\mathbf{u}}$. Parametrize the new solution by

$$
\underline{\mathbf{u}}_{\text {new }}=\underline{\mathbf{u}}_{\text {old }}+\lambda \delta \underline{\mathbf{u}}
$$


and determine the parameter $\lambda>0$ such that the residual of the new solution,

$$
\left\|\mathcal{S}\left(\underline{\mathbf{u}}_{\text {new }}\right)\right\|,
$$

has sufficiently decreased. Of course, close enough to the true solution, the full Newton-Raphson step $\lambda=1$ will lead to quadratic convergence. PETSc offers different algorithms to perform this line-search. The line search ensures that in each iteration the residual does indeed decrease, which is not guaranteed in NewtonRaphson without line searches.

\subsubsection{Linear Solve}

In each Newton-Raphson iteration one has to solve Eq. (42), a linear system of $N_{D F}$ equations. For large systems of linear equations, iterative linear solvers [5] are most efficient. Such iterative solvers require solely the ability to compute the matrix-vector product $\mathcal{J} \underline{\mathbf{v}}$ for a given vector $\underline{\mathbf{v}}$. Since spectral derivatives and spectral interpolation lead to full (i.e. non-sparse) matrices it is impractical to set up the matrix $\mathcal{J}$ explicitly. One can compute these matrix-vector products instead with the linearized variant of the code that computes the operator $\mathcal{S}$, i.e. equations (38a)-(38g) and their multidimensional generalizations. Thus our method requires the linearizations of the operator $\mathcal{N}$ [Eq. (38a)] and of the boundary conditions [Eqs. (38b) and (38d)]. The matching equations (38d)-(38g) are linear anyway, so one can reuse code from $\mathcal{S}$ for these equations. The linearizations are merely Frechet derivatives [6] of the respective operators evaluated at the collocation points, and therefore the Newton-Raphson iteration applied to the discretized equations is equivalent to the Newton-Kantorovitch iteration applied to the PDE.

PETSc includes several different linear iterative solvers (GMRES, TFQR, ...) that can be employed for the linear solve inside the Newton-Raphson iteration. The choice of linear solver and of options for the linear solver and for the NewtonRaphson iteration are made at runtime. This allows one to experiment with different linear solvers and with a variety of options to find an efficient combination. Note that the matching conditions (18) and (19) lead to a nonsymmetric matrix $\mathcal{J}$. Therefore only iterative methods that allow for nonsymmetric matrices can be used.

\subsubsection{Preconditioning}

In practice one will find that the Jacobian $\mathcal{J}$ is ill-conditioned and thus the iterative method employed to solve Eq. (42) will need an increasing number of iterations as the number of collocation points is increased. The spectral condition number $\kappa$ of a matrix is the ratio of largest to smallest eigenvalue of this matrix,

$$
\kappa=\frac{\lambda_{\max }}{\lambda_{\min }} .
$$

For second order differential equations discretized with Chebyshev polynomials, one finds $\kappa \propto N^{4}, N$ being the number of grid points per dimension. Solving a linear system to given accuracy will require 2, 5] $\mathcal{O}(\kappa)$ iterations of the Richardson method, and $\mathcal{O}(\sqrt{\kappa})$ iterations of modern iterative methods like conjugate gradients or GMRES. Although modern methods are better than Richardson iteration, it is still vital to keep $\kappa$ close to 1 . 
This is achieved with preconditioning. Instead of solving Eq. (42) directly, one solves

$$
\mathcal{B J} \delta \underline{\mathbf{u}}=-\mathcal{B} \underline{\mathbf{r}},
$$

with the preconditioning matrix $\mathcal{B}$. Now the iterative solver deals with the matrix $\mathcal{B J}$. If $\mathcal{B}$ is a good approximation to $\mathcal{J}^{-1}$, then $\mathcal{B J}$ will be close to the identity matrix, the condition number will be close to unity, and the linear solver will converge quickly.

Hence the problem reduces to finding a matrix $\mathcal{B}$ that approximates $\mathcal{J}^{-1}$ sufficiently well and that can be computed efficiently. There exist many different approaches, most notably finite difference preconditioning 22 and finite element preconditioning 12]; we will follow a two-stage process proposed by Orszag 22. First, initialize a matrix $\mathcal{A}_{F D}$ with a finite difference approximation of the Jacobian $\mathcal{J}$. Second, approximately invert $\mathcal{A}_{F D}$ to construct $\mathcal{B}$,

$$
\mathcal{B} \approx \mathcal{A}_{F D}^{-1}
$$

In one spatial dimension $\mathcal{A}_{F D}$ is tridiagonal and direct inversion $\mathcal{B} \equiv \mathcal{A}_{F D}^{-1}$ is feasible. In two or more dimensions, direct inversion of $\mathcal{A}_{F D}$ is too expensive; for problems in one two-dimensional subdomain, hardcoded incomplete LU-factorizations have been developed[B]. In our case we have to deal with the additional complexity that the Jacobian and therefore $\mathcal{A}_{F D}$ contains matching conditions. Since we choose the domain decomposition at runtime, nothing is known about the particular structure of the subdomains.

We proceed as follows: We initialize $\mathcal{A}_{F D}$ with the finite difference approximation of $\mathcal{J}$. It is sufficient to include those terms of the Jacobian in $\mathcal{A}_{F D}$ that cause the condition number to increase with the expansion order. These are the second spatial derivatives and the first derivatives from matching conditions and boundary conditions, Eqs. (38d) and (38d). Including the value matching conditions (38d), (381), (38g) in $\mathcal{A}_{F D}$ improves the ability of the preconditioner to represent modes extending over several subdomains and thus decreases the number of iterations, too. In the first example in section 4.1 we demonstrate that preconditioning is indeed necessary, and that one should precondition not only the second order derivatives, but also the matching conditions. Some details about the finite difference approximations are given in appendix A.

Having set up $\mathcal{A}_{F D}$ we then use the software package PETSc 4 for the approximate inversion of Eq. (47). PETSc provides many general purpose preconditioners that perform the step (47) either explicitly or implicitly, most notably ILU and the overlapping Schwarz method. With PETSc we can explore these to find the most efficient one. We will describe our particular choices for preconditioning below for each example.

\section{5. $\mathcal{S}$ in higher dimensions}

Generalizing $\mathcal{S}$ to multiple dimensions is conceptually straightforward, since Eqs. (38) generalize nicely to higher dimensions. In order to simplify the matching between touching subdomains, we require that on a surface shared by touching subdomains, the collocation points are identical. If, for example, two three-dimensional rectangular blocks touch along the $x$-axis, then both blocks must have identical lower and upper bounds of the blocks along the $y$ and $z$ axis and both blocks must 
use the same mappings and the same number of collocation points along the $y$ - and $z$-axis. For concentric spherical shells, this restriction implies that all concentric shells must have the same number of collocation points in the angular directions. With this restriction, matching between touching subdomains remains a point-bypoint operation.

For overlapping domains, no restriction is needed. If a boundary point of one subdomain happens to be within another subdomain, then an equation analogous to (38f) is enforced using spectral interpolation.

The actual implementation of the operator $\mathcal{S}$ involves bookkeeping to keep track of which subdomains overlap or touch, or of what equation to enforce at which grid point. When running in parallel, matching conditions have to be communicated across processors. We utilize the software package KeLP[3], which provides functionality to iterate over boundary points of a specific subdomain. It also provides routines to handle the interprocessor communication needed for the matching conditions.

\subsection{Extension of $\mathcal{S}$ to Spherical Shells}

Spherical shells have the additional complexity of having more collocation points than spectral coefficients, $N_{c o l}>N_{\text {spec }}$, at least in our formulation. Transforming to spectral space and back to real space projects the real-space values into a $N_{\text {spec-dimensional subspace. Since spectral transforms are used for derivatives and }}$ interpolation, a sphere has effectively only $N_{\text {spec }}$ degrees of freedom. If we naively try to impose $N_{c o l}$ equations, one at each collocation point, and if we try to solve for real space values at each collocation point, we find that the linear solver does not converge. This happens because more equations are imposed than degrees of freedom are available. Thus we cannot solve for the real space values $u_{i j k}$ in a spherical shell.

The next choice would be to solve for the spectral coefficients $\tilde{u}_{k l m}$ as defined in Eq. (32) This is also problematic as it prohibits finite-difference preconditioning. One finds guidance on how to proceed by considering the prototypical elliptic operator, the Laplacian. Application of $\nabla^{2}$ to an expansion in spherical harmonics yields

$$
\nabla^{2} \sum_{l, m} a_{l m}(r) Y_{l m}=\sum_{l, m}\left[-\frac{l(l+1) a_{l m}(r)}{r^{2}}+\frac{1}{r^{2}} \frac{\partial}{\partial r}\left(r^{2} \frac{\partial a_{l m}(r)}{\partial r}\right)\right] Y_{l m} .
$$

We see that the different $(l m)$-pairs are uncoupled. The angular derivatives will therefore be diagonal in spectral space (with diagonal elements $-l(l+1) / r^{2}$ ). However, one has to precondition the radial derivatives in order to keep the spectral conditioning number low and must therefore keep real-space information about the radial direction. We therefore solve for the coefficients $\hat{u}_{i l m}$ of an expansion defined by

$$
u\left(r_{i}, \theta, \phi\right)=\sum_{l=0}^{L} \sum_{m=-l}^{l} \hat{u}_{i l m} Y_{l m}(\theta, \phi) .
$$

This mixed real/spectral expansion has $N_{\text {spec }}$ coefficients $\hat{u}_{i l m}$ and retains real space information about the radial coordinate necessary for finite difference preconditioning. In order to precondition the flat space Laplacian in a spherical shell, 

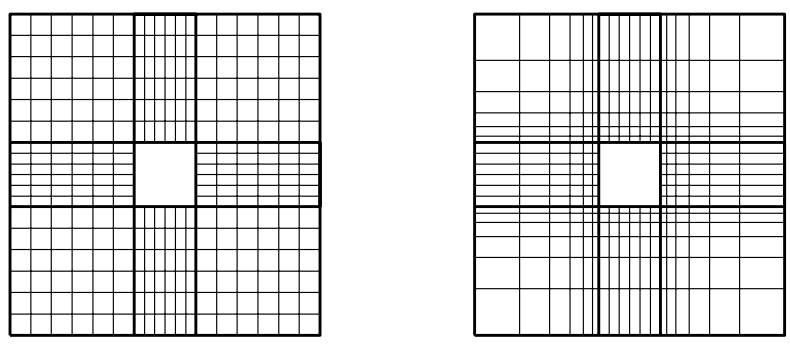

FIG. 2 Domain decomposition for Laplace equation in a square. The left plot illustrates linear mappings in all subdomains and the right plot shows log-linearlog mappings along each axis.

$\mathcal{A}_{F D}$ is initialized with the diagonal matrix $\operatorname{diag}\left(-l(l+1) / r_{i}^{2}\right)$ for the angular piece of $\nabla^{2}$ and with finite differences for the radial derivatives. More general differential operators are discussed in the last example, section 1.3 , and in appendix B.

In order to evaluate $\mathcal{S} \underline{\mathbf{u}}$ for a spherical shell, we proceed as follows. $\underline{\mathbf{u}}$ contains the coefficients $\hat{u}_{i l m}$. Transform these coefficients to real space values. This involves only an angular transform. Compute boundary conditions, matching conditions, and the residual of the nonlinear elliptic operator $\mathcal{N}$ at each collocation point as in rectangular blocks. At this stage we have $N_{c o l}$ collocation point values, all of which should vanish for the desired solution. We transform these values back into the coefficients of Eq. (49) and return these coefficients as the residual of the operator $\mathcal{S}$.

\section{EXAMPLES}

\section{1. $\quad \nabla^{2} u=0$ in $2-\mathrm{D}$}

As a first test, we solve the Laplace equation in two dimensions with Dirichlet boundary conditions:

$$
\begin{aligned}
\nabla^{2} u(x, y) & =0 & (x, y) & \in \mathcal{D} \\
u(x, y) & =\ln \left(x^{2}+y^{2}\right) & (x, y) & \in \partial \mathcal{D}
\end{aligned}
$$

The computational domain $\mathcal{D}$ is a square with side $2 L$ centered on the origin with a smaller square with side 2 excised:

$$
\mathcal{D}=\{(x, y) \mid-L \leq x, y \leq L\}-\{(x, y) \mid-1<x, y<1\}
$$

This domain is decomposed into 8 touching rectangles as shown in figure 2. This figure also illustrates the difference between linear mappings and logarithmic mappings. The right plot of figure 2 shows that logarithmic mappings move grid points closer to the excised rectangle. For clarity, both plots neglect the fact that the Chebyshev collocation points given in Eq. (8) are clustered toward the boundaries.

We solve Eqs. (50) and (51) for three cases:

- $L=5$ with linear mappings

- $L=100$ with linear mappings 


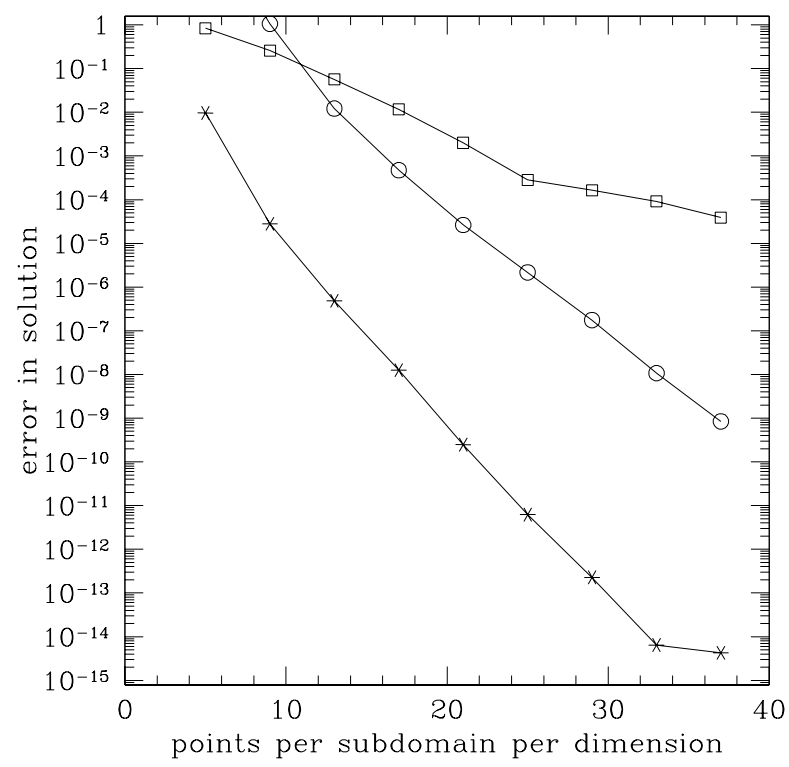

FIG. 3 rms-errors of solution of Laplace equation in a square. Stars denote $L=5$ with linear mappings, squares $L=100$ with linear mappings, and circles $L=100$ with log mappings.

- $L=100$ with logarithmic mappings.

Equation (50) is linear, therefore only one Newton-Raphson iteration with one linear solve is necessary. The numerical solution is compared to the analytic solution $u(x, y)=\ln \left(x^{2}+y^{2}\right)$. The errors are shown in figure 3. In the small computational domain extending only to $x, y= \pm 5$, the accuracy of the solution quickly reaches numerical roundoff. In the larger domain extending to $L=100$ the achievable resolution with the same number of collocation points is of course lower. With linear mappings we achieve an absolute accuracy of $10^{-4}$ with a total of $\sim 10000$ collocation points. This is already better than finite difference codes. However this accuracy can be increased with better adapted mappings. Since the solution $\ln \left(x^{2}+y^{2}\right)$ changes much faster close to the origin than far away, one expects better convergence if more collocation points are placed close to the origin. This can be achieved with logarithmic mappings. Figure 3 shows that logarithmic mappings roughly double the convergence rate. At the highest resolution the difference is over four orders of magnitude.

Table 1 compares the number of iterations $N_{i t s}$ in the linear solver for different choices of the finite difference preconditioning matrix $\mathcal{A}_{F D}$ [section 3.4.3]. Without any preconditioning, $\mathcal{A}_{F D}=1, N_{i t s}$ increases very quickly with the number of collocation points. If only second derivative terms are included in $\mathcal{A}_{F D}$ then $N_{i t s}$ grows more slowly. The number of iterations becomes almost independent of resolution if both second derivatives and the matching conditions (38d) and (38e) are incorporated into $\mathcal{A}_{F D}$. In this case ILU(2) preconditioning completely controls the largest eigenvalue $\lambda_{\max }$, and it is then the smallest eigenvalue $\lambda_{\min }$ that causes the number of iterations to increase. It is typical that ILU has difficulties 


\section{TABLE 1}

Number of iterations $N_{i t s}$ in the linear solver for various kinds of preconditioning as a function of the number of collocation points $N$ per dimension per subdomain. no $P C$ stands for no preconditioning at all, $P C \nabla^{2}$ includes only second derivative terms in $\mathcal{A}_{F D}$, whereas full $P C$ includes also matching conditions. ILU(2) decomposition, exact and approximate inversion of $\mathcal{A}_{F D}$ are explored.

\begin{tabular}{|c|c|c|c|c|c|c|c|c|c|}
\hline \multirow{3}{*}{$N$} & \multirow{3}{*}{$\begin{array}{c}\text { no PC } \\
N_{i t s}\end{array}$} & \multicolumn{4}{|c|}{$\mathcal{B}=\operatorname{ILU}(2)\left[\mathcal{A}_{F D}\right]$} & \multirow{2}{*}{\multicolumn{3}{|c|}{$\begin{array}{c}\mathcal{B}=\mathcal{A}_{F D}^{-1} \\
\text { full } \mathrm{PC}\end{array}$}} & \multirow{3}{*}{$\begin{array}{c}\mathcal{B} \approx \mathcal{A}_{F D}^{-1} \\
\text { full PC } \\
N_{i t s}\end{array}$} \\
\hline & & $\mathrm{PC} \nabla^{2}$ & & full $\mathrm{P}$ & & & & & \\
\hline & & $N_{i t s}$ & $N_{i t s}$ & $\lambda_{\max }$ & $\lambda_{\min }$ & $N_{i t s}$ & $\lambda_{\max }$ & $\lambda_{\min }$ & \\
\hline 5 & 54 & 22 & 7 & 2.1 & 0.96 & 4 & 1.7 & 1.00 & 4 \\
\hline 9 & 194 & 38 & 10 & 2.2 & 0.64 & 6 & 2.0 & 1.00 & 6 \\
\hline 13 & 302 & 49 & 11 & 2.3 & 0.35 & 6 & 2.2 & 1.00 & 6 \\
\hline 17 & 594 & 64 & 14 & 2.4 & 0.20 & 6 & 2.2 & 1.00 & 6 \\
\hline 21 & 967 & 109 & 17 & 2.4 & 0.13 & 6 & 2.4 & 1.00 & 6 \\
\hline 25 & 1244 & 140 & 20 & 2.5 & 0.09 & 6 & 2.4 & 1.00 & 7 \\
\hline 29 & & 206 & 25 & 2.6 & 0.05 & 6 & 2.5 & 1.00 & 7 \\
\hline 33 & & 255 & 27 & 2.6 & 0.04 & 7 & 2.4 & 1.00 & 7 \\
\hline
\end{tabular}

controlling the long wavelength modes, and the problem is aggravated because the subdomains are only weakly coupled. Table 1 also contains results for exact inversion of $\mathcal{A}_{F D}$. Exact inversion controls $\lambda_{\text {min }}$, too, and now the number of iterations is independent of resolution. However, direct inversion is an expensive process and only possible for small problems like this one. Finally, the table also contains the iteration count for approximate inversion of $\mathcal{A}_{F D}$, which is our preferred method for more complex geometries in 3 dimensions. It will be explained in detail in the next example. The eigenvalues in table 1 are estimates obtained by PETSc during the linear solve.

\subsection{Quasilinear Laplace equation with two excised spheres}

This solver was developed primarily for elliptic problems in numerical relativity. Accordingly we now solve an equation that has been of considerable interest in that field over the last few years (see e.g. [10, 9] and references therein). Readers not familiar with relativity can simply view this problem as another test example for our new solver円. We solve

$$
\nabla^{2} \psi+\frac{1}{8} A^{2} \psi^{-7}=0
$$

for the function $\psi=\psi(x, y, z) . A^{2}=A^{2}(x, y, z)$ is a known, positive function, and the computational domain is $\mathbb{R}^{3}$ with two excised spheres,

$$
\mathcal{D}=\mathbb{R}^{3}-S_{1}-S_{2} .
$$

\footnotetext{
${ }^{1}$ The solution of this problem describes two black holes. The surfaces of the spheres $S_{1,2}$ correspond to the horizons of the black holes, the function $A^{2}$ encodes information about spins and velocities of the black holes, and the solution $\psi$ measures the deviation from a flat spacetime. Far away from the black holes one has $\psi \approx 1$ with an almost Minkowski space, close to the holes we will find $\psi \sim 2$ with considerable curvature of spacetime.
} 


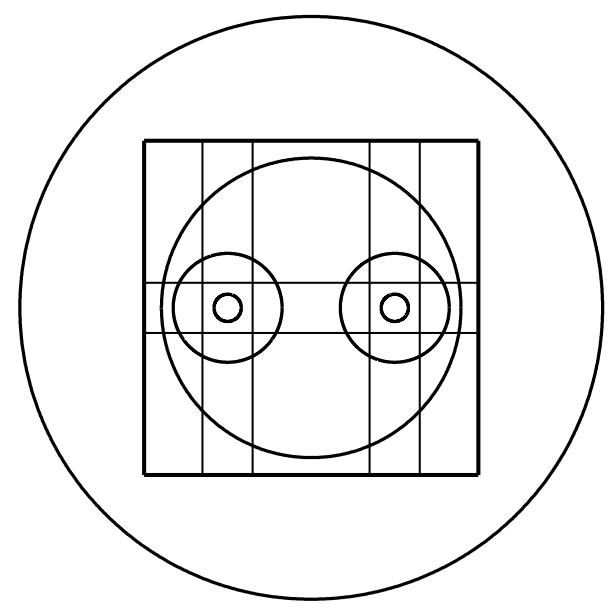

FIG. 4 Cut through the domain decomposition for the computational domain (54).

The radii $r_{1 / 2}$ and centers of the spheres are given. The function $\psi$ must satisfy a Dirichlet boundary condition at infinity, and Robin boundary conditions at the surface of each excised sphere:

$$
\begin{aligned}
\psi & \rightarrow 1 & & \text { as } r \rightarrow \infty \\
\frac{\partial \psi}{\partial r}+\frac{\psi}{2 r_{i}} & =0 & & \vec{r} \in \partial S_{i}, \quad i=1,2
\end{aligned}
$$

$\partial / \partial r$ in Eq. (56) denotes the radial derivative in a coordinate system centered at the center of sphere $i$.

Figure 1 sketches the domain decomposition used for the computational domain $\mathcal{D}$. We surround each excised sphere with a spherical shell. These two spherical shells are matched together with $5 \times 3 \times 3$ rectangular blocks, where the two blocks that contain the excised spheres $S_{1,2}$ are removed. Finally, we surround this structure with a third spherical shell extending to very large outer radius. This gives a total of 46 subdomains, namely 3 shells and 43 rectangular blocks.

In the inner spheres we use a log mapping for the radial coordinate. In the rectangular blocks, a combination of linear and logarithmic mappings is used similar to the 2D example in figure 2. In the outer sphere an inverse mapping is used which is well adapted to the fall-off behavior $\psi \sim 1+a r^{-1}+\cdots$ for large radii $r$. The outer radius of the outer spherical shell is chosen to be $10^{9}$ or $10^{10}$ and a Dirichlet boundary condition $\psi=1$ is used to approximate Eq. (55).

We now present two solutions with different sizes and locations of the excised spheres. In sections 4.2.3 to 4.2.6, we then discuss several topics including preconditioning and parallelization.

\subsubsection{Equal sized spheres}

First we choose two equal sized spheres with radii $r_{1}=r_{2}=1$. The separation between the centers of the spheres is chosen to be 10 , the outer radius of the outer 


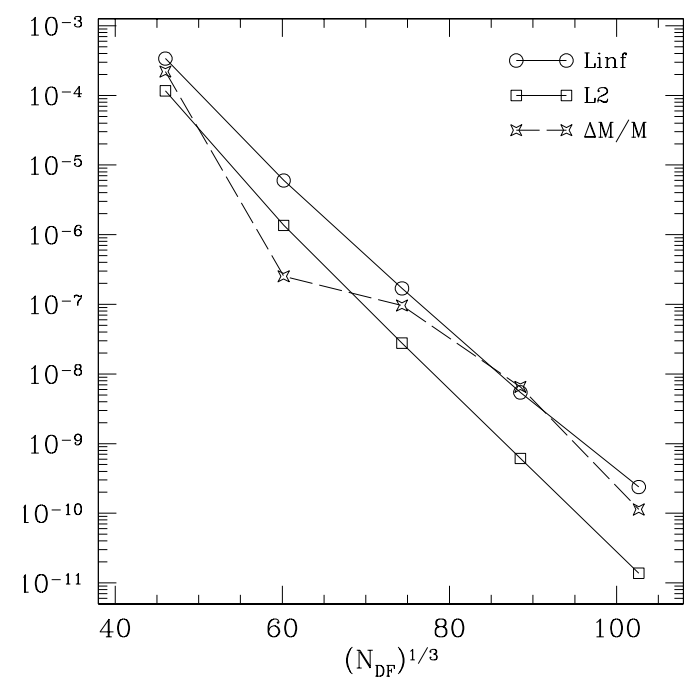

FIG. 5 Convergence of solution of (53)-(56) with radii of excised spheres $r_{1}=r_{2}=$ 1 at separation 10. $N_{D F}, L_{i n f}, L_{2}, M$ and $\Delta M$ are defined in the text immediately before and after Eq. (57).

sphere is $10^{9}$.

We solve equation (53) at several resolutions. The highest resolution uses $29^{3}$ collocation points in each rectangular block, $29 \times 21 \times 42$ collocation points (radial, $\theta$ and $\phi$ directions) in the inner spherical shells and $29 \times 16 \times 32$ in the outer spherical shell. We use the difference in the solutions at neighboring resolutions as a measure of the error. We denote the pointwise maximum of this difference by $L_{\text {inf }}$ and the root-mean-square of the grid point values by $L_{2}$. We also compute at each resolution the quantity

$$
M=-\frac{1}{2 \pi} \int_{\infty} \frac{\partial \psi}{\partial r} d^{2} S
$$

which is the total mass of the binary black hole system. $M$ will be needed in the comparison to a finite difference code below. The difference $\Delta M$ between $M$ at neighboring resolutions is again a measure of the error of the solution.

Figure 5 shows the convergence of the solution $\psi$ with increasing resolution. Since the rectangular blocks and the spheres have different numbers of collocation points, the cube root of the total number of degrees of freedom, $N_{D F}^{1 / 3}$ is used to label the $x$-axis. The exponential convergence is apparent. Because of the exponential convergence, and because Linf, L2 and $\Delta M$ utilize differences to the next lower resolution, the errors given in figure 5 are essentially the errors of the next lower resolution. Note that at the highest resolutions the approximation of the outer boundary condition (55) by a Dirichlet boundary condition at finite outer radius $10^{9}$ becomes apparent: If we move the outer boundary to $10^{10}, M$ changes by $2 \cdot 10^{-9}$ which is of order $1 / 10^{9}$ as expected.

On the coarsest resolution $\psi=1$ is used as the initial guess. Newton-Raphson then needs six iterations to converge. On the finer resolutions we use the result of the previous level as the initial guess. These initial guesses are so good that one 


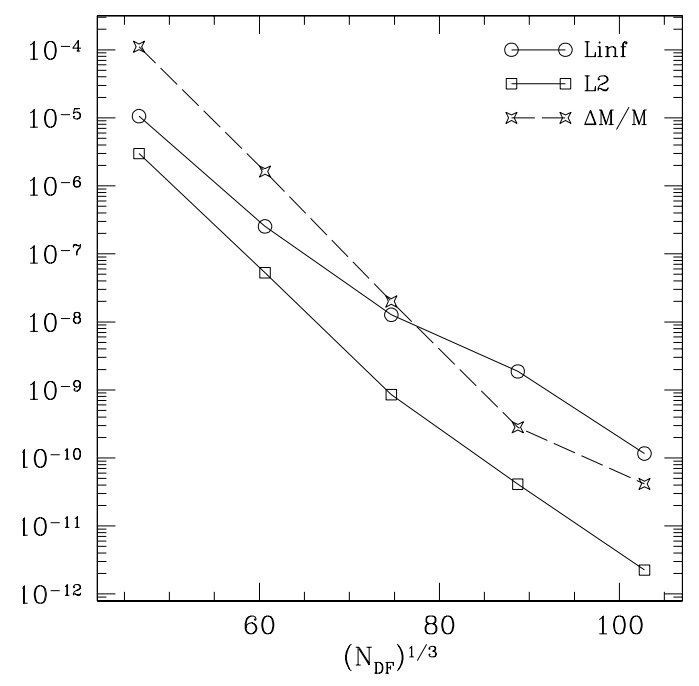

FIG. 6 Convergence of solution of (53)-(56) for excised spheres of radii $r_{1}=1$, $r_{2}=0.05$ at separation 100. Symbols as in figure 5 .

Newton-Raphson iteration is sufficient on each resolution.

\subsubsection{Nonequal spheres - Different length scales}

With the multidomain spectral method it is possible to distribute resolution differently in each subdomain. This allows for geometries with vastly different length scales. As an example, we again solve equations (53)-(56). The radii of the spheres are now $r_{1}=1$ and $r_{2}=0.05$, and the separation of the centers of the spheres is 100. The separation of the holes is thus 2000 times the radius of the smaller sphere. A finite difference code based on a Cartesian grid for this geometry would have to use adaptive mesh refinement.

With the spectral solver, we still use the domain decomposition depicted in figure 1 , but now the inner radii of the two inner spherical shells are different. The outer boundary of the outer sphere is at $10^{10}$. The number of collocation points in each subdomain is almost identical to the case with equal sized spheres of figure 5 , except we add 8 additional radial collocation points to the shell around the small excised sphere. As before we solve on different resolutions and compute the norms of the differences of the solution between different resolutions, as well as of the total mass $M$. The results are shown in figure 6. The exponential convergence shows that the solver can handle the different length scales involved in this problem.

\subsubsection{Preconditioning}

The finite difference approximation $\mathcal{A}_{F D}$ is initialized with the second derivative terms, the matching conditions in touching domains [cf. Eqs. (38d) and (38d)], and with a FD approximation of the Robin boundary condition Eq. (56). Running on a single processor, we could again define the preconditioner $\mathcal{B}$ via an ILU decomposition of $\mathcal{A}_{F D}$. However, when running on multiple processors, an ILU decomposition requires a prohibitive amount of communication, and block ASM 
preconditioning 27] with one block per processor becomes favorable. After considerable experimentation, we settled on an implicit definition of $\mathcal{B}$ via its action on vectors. $\mathcal{B} \underline{\mathbf{v}}$ is defined to be the approximate solution $\underline{\mathbf{w}}$ of

$$
\mathcal{A}_{F D \underline{\mathbf{w}}}=\underline{\mathbf{v}} \text {. }
$$

Equation (58) is solved using a second, inner iterative solver, usually GMRES preconditioned with ILU (on a single processor) or a block ASM method (on multiple processors). The inner solver is restricted to a fixed number of iterations. Applying a fixed number of iterations of an iterative solver is not a linear operation, hence $\mathcal{B}$ represents no longer a matrix, but a nonlinear operator. In the outer linear solve we therefore use FGMRES[26, a variant of GMRES that does not require that the preconditioner $\mathcal{B}$ is linear. With this preconditioning the outer linear solver needs about 20 iterations to reduce the residual of the linear solve by $10^{-5}$.

More inner iterations reduce the number of iterations in the outer linear solve, but increase the computations per outer iteration. We found the optimal number of inner iterations to be between 15-20. In all the computations given in this paper we use 20 inner iterations, except for the 2-D example in table 1 where 10 inner iterations sufficed.

\subsubsection{Multigrid}

We also experimented with multigrid algorithms 8 , 25 to improve the runtime. The potential for multigrid is fairly small, since the number of collocation points is so low. In this particular problem, an accuracy of better than $10^{-6}$ can be achieved with $17^{3}$ grid points per domain, which limits multigrid to at most two coarse levels.

In addition it is not trivial to construct a restriction operator. The obvious and canonical choice for a restriction operator is to transform to spectral space, discard the upper half of the spectral coefficients, and transform back to real space on a coarser grid. This does not work here because the operator $\mathcal{S}$ uses the boundary points of each subdomain to convey information about matching between subdomains and about boundary conditions. Since these boundary points are filled using different equations than the interior points, the residual will typically be discontinuous between boundary points of a subdomain and interior points. Information about discontinuities is mainly stored in the high frequency part of a spectral expansion and discarding these will thus result in a loss of information about matching between grids. However, the coarse grid correction of a multigrid algorithm is supposed to handle long wavelength modes of the solution. In our case these extend over several subdomains and thus information about matching is essential. Hence the simple approach of discarding the upper half of the frequencies discards the most vital parts of the information required by the coarse grid solver.

Thus one seems to be compelled to use a real space restriction operator. We examined straight injection 25 which performed fairly well. The execution speed was comparable to the preconditioning with an inner linear solve as described in section 4.2.3. Since we did not achieve a significant code speed-up, there was no reason to keep the increased complexity of the multigrid algorithm.

\subsubsection{Comparison to a Finite Difference Code}

The computational domain Eq. (54) is challenging for standard finite difference codes based on a regular Cartesian grids for two reasons: 


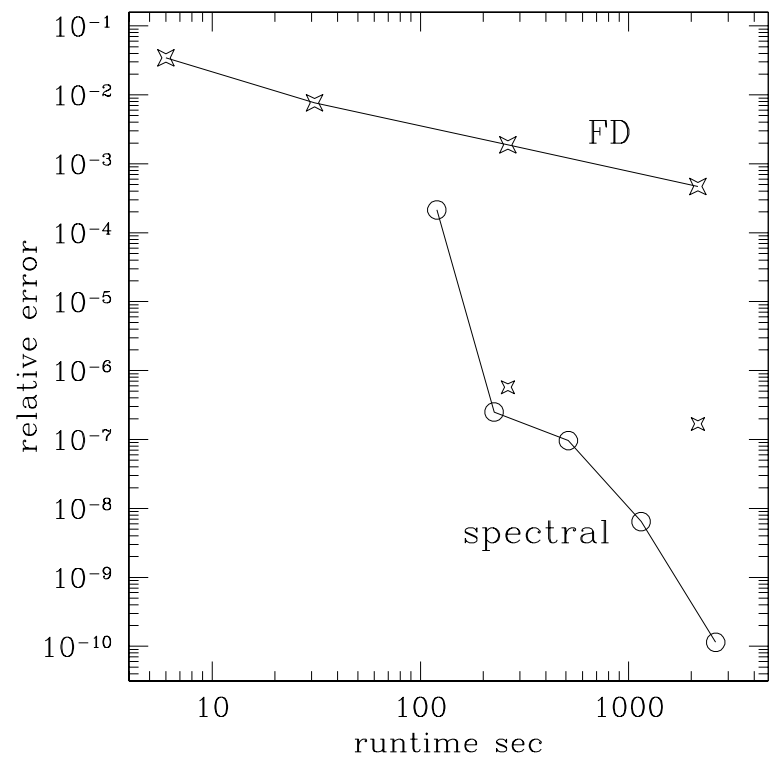

FIG. 7 Comparison of runtime vs. achieved accuracy for the new spectral solver and the highly optimized Cadéz code. Plotted is the achieved accuracy of the total mass $M$ vs. runtime needed to solve Eqs. (53)-(56) for both codes.

1. The boundaries of the excised spheres do not coincide with coordinate boundaries, so complicated interpolation/extrapolation is needed to satisfy the boundary condition (56) (This problem led to a reformulation of the underlying physical problem without excised spheres[河).

2. Resolving both the rapid changes close to the excised spheres and the fall-off behavior toward infinity requires a large number of grid points.

In 10 three different methods were developed to solve the boundary value problem (53)-(56). The best one turned out to be a finite difference code based on a specially adapted coordinate system, the so-called Cadéz coordinates. This code is an FAS-multigrid algorithm developed specifically for the differential equation (53). Care was taken that the truncation error is strictly even in grid-spacing $h$, thus allowing one to take two or three solutions at different resolutions and Richardson extrapolate to $h \rightarrow 0$. The Cadéz code is thus specially built for this equation in this geometry and it is unlikely that it can be significantly improved upon by any finite difference method.

On the other hand, our spectral solver is general purpose. The domain decomposition is not restricted to $\mathbb{R}^{3}$ with two excised spheres and we do not employ any specific optimizations for this particular problem.

We compare these two codes for the configuration with equal sized spheres. Figure 7 shows a plot of runtime vs. achieved accuracy for both codes. These runs were performed on a single RS6000 processor; the highest resolution runs needed about 1GB of memory. The solid line labeled FD represents the results of the finite difference code without Richardson extrapolation. This line converges quadratically in grid spacing. The two stars represent Richardson extrapolated 
values. The superiority of the spectral code is obvious. In accuracy, the spectral method outperforms even the finite difference code with Richardson extrapolation by orders of magnitude. Only very few finite difference codes allow for Richardson extrapolation, hence one should also compare the finite difference code without Richardson extrapolation to the spectral code: Then the lowest resolution of the spectral code is as accurate as the highest resolution of the finite difference code and faster by a factor of 20 . Note also that the Cadéz code cannot handle excised spheres of very different sizes or spheres that are widely separated. In particular, it cannot be used for the configuration in section 4.2.2, which is readily solved by our method.

\subsubsection{Parallelization}

Most computations during a solve are local to each subdomain; the operator $\mathcal{S}$ and the Jacobian $\mathcal{J}$ need communicate only matching information across subdomains. The inner linear solve is a completely standard parallel linear solve with an explicitly known matrix $\mathcal{A}_{F D}$. The software package PETSc has all the necessary capabilities to solve this system of equations efficiently in parallel. Hence parallelization by distributing different subdomains to different processors is fairly straightforward.

However, different elements of the overall solve scale with different powers of the number of collocation points per dimension. If we denote the number of collocation points per dimension by $N$, the following scalings hold in three dimensions (the most interesting case): A spectral transform in a rectangular domain requires $\mathcal{O}\left(N^{3} \log N\right)$ operations; the transform in a sphere - where no useful fast transform for the Legendre polynomials is available - requires $\mathcal{O}\left(N^{4}\right)$ operations; interpolation to one point is $\mathcal{O}\left(N^{3}\right)$, so interpolation to all $\mathcal{O}\left(N^{2}\right)$ boundary points scales like $\mathcal{O}\left(N^{5}\right)$. Thus the optimal assignment of subdomains to processors is a function of $N$. Moreover, assignment of subdomains to processors is a discrete process - it is not possible to move an arbitrary fraction of computations from one processor to the another. One always has to move a whole subdomain with all the computations associated with it. This makes efficient load balancing difficult.

At high resolution, the $\mathcal{O}\left(N^{5}\right)$ interpolation consumes most of the runtime. Note that the outer spherical shell interpolates to 78 block surfaces, whereas the inner shells each interpolate to 6 block surfaces. These interpolations are parallelized by first distributing the data within each sphere to all processors. Then each processor interpolates a fraction of the points and the results are gathered again.

We present scaling results in table 2. These results were obtained on the SP2 of the physics department of Wake Forest University, and on NCSA's Platinum cluster, whose nodes have two Intel Pentium processors each. The listed times are cumulative times for solving at five different resolutions, each solve using the next lower solution as initial guess. Not included in these times is the set up in which the code determines which subdomain is responsible for interpolating which "overlapping" boundary point. Also not included is input/output.

On the SP2 we achieve a scaling efficiency of $75 \%$, whereas the Intel cluster has a lower scaling efficiency between around 54\% (8 processors), and $41 \%$ (46 processors). Given all the limitations mentioned above these numbers are very encouraging.

Changing from serial to parallel execution degrades performance in two ways: First, the ILU preconditioner used within the approximate inner linear solve is 
TABLE 2

Runtime and scaling efficiency. Three processors host one shell and $n_{1}$ blocks each, the remaining processors host $n_{2}$ blocks each. The last four columns refer to the Platinum cluster.

\begin{tabular}{|c|cc|rc|rr|rr|}
\hline & & & \multicolumn{2}{|c|}{ SP2 } & \multicolumn{2}{|c|}{2 procs/node } & \multicolumn{2}{|c|}{ proc/node } \\
Nprocs & $n_{1}$ & $n_{2}$ & $\mathrm{t}[\mathrm{sec}]$ & eff. & $\mathrm{t}[\mathrm{sec}]$ & eff. & $\mathrm{t}[\mathrm{sec}]$ & eff. \\
\hline 1 & & & 2344 & & 1654 & & 1654 & \\
4 & 10 & 13 & 786 & 0.75 & 764 & 0.54 & 643 & 0.64 \\
8 & $4-5$ & 6 & 384 & 0.76 & 381 & 0.54 & 304 & 0.68 \\
18 & 0 & 3 & & & 198 & 0.46 & 156 & 0.59 \\
26 & 0 & 2 & & & 140 & 0.45 & 111 & 0.57 \\
46 & 0 & 1 & & & 87 & 0.41 & 73 & 0.49 \\
\hline
\end{tabular}

replaced by an overlapping block ASM preconditioner. Since this preconditioner is less efficient than ILU, the approximate inner linear solve is less accurate after its fixed number of iterations. Therefore the outer linear solve needs more iterations to converge to the required accuracy of $10^{-5}$. The single processor code needs 19 outer linear iterations, whereas the parallel codes need 23 or 24 . Thus the maximally achievable scaling efficiency is limited to $19 / 23 \approx 0.83$. The scaling efficiency on the SP2 is close to this limit.

The second reason for low scaling efficiency is that we have not optimized the MPI calls in any way. The fact that the scaling efficiency on the cluster is much better if only one processor per node is used, suggests that the MPI calls are a bottleneck. Using both processors on a node doubles the communication load on that node which doubles the waiting time for MPI communication. The higher scaling efficiency on the SP2 which has faster switches also suggests that the runs on the PC cluster are communication limited.

\subsection{Coupled PDEs in nonflat geometry with excised spheres}

So far we have been considering only PDEs in a single variable. However, the definition of the operator $\mathcal{S}$ is not restricted to this case. In this section we present a solution of four coupled nonlinear PDEs. These equations are

$$
\begin{aligned}
\tilde{\nabla}^{2} \psi-\frac{1}{8} \psi \tilde{R}-\frac{1}{12} \psi^{5} K^{2}+\frac{1}{8} \psi^{-7} \sum_{i, j=1}^{3} \tilde{A}_{i j} \tilde{A}^{i j}=0 & \\
\tilde{\Delta}_{L} V^{i}-\frac{2}{3} \psi^{6} \tilde{\nabla}^{i} K+\sum_{j=1}^{3} \tilde{\nabla}_{j} \tilde{M}^{i j}=0, & i=1,2,3
\end{aligned}
$$

These equations are important for the binary black hole problem. The exact definitions of the various terms can be found in 23 . For this paper, only the following information is necessary: $\tilde{\nabla}^{2}$ is the Laplace operator on a nonflat three-dimensional manifold, hence Eq. (59) is an elliptic equation for $\psi \cdot \tilde{\Delta}_{L}$ is a variant of the vector Laplacian, thus Eq. (60) is an elliptic equation for the vector $V^{i}, i=1,2,3$. The variables $\tilde{A}_{i j}$ and $\tilde{A}^{i j}$ are functions of $V^{i}$, so that Eqs. (59) and (60) have to be solved simultaneously. The functions $\tilde{R}, K$ and $\tilde{M}^{i j}$ are given. 


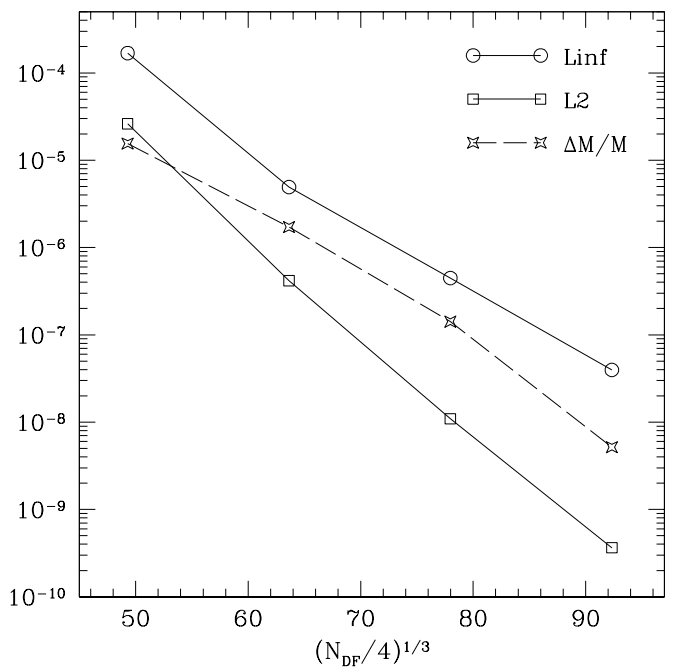

FIG. 8 Convergence of solution to coupled PDEs (59) and (60). Definitions as in figure 5 .

The computational domain again has two spheres $S_{1,2}$ excised,

$$
\mathcal{D}=\mathbb{R}^{3}-S_{1}-S_{2} .
$$

The radii of the excised spheres are $r_{1}=r_{2}=2$ and the separation between the centers is 10 .

We have Dirichlet boundary conditions on all boundaries:

$$
\begin{aligned}
\psi & =1, \\
V^{i} & =0, \quad i=1,2,3 .
\end{aligned}
$$

We solve the problem again at several different resolutions. On the coarsest level two Newton-Raphson iterations are necessary, whereas the finer levels need only one Newton-Raphson iteration. The linear solver needs 30 iterations to reduce the residual by $10^{5}$. Details about constructing $\mathcal{A}_{F D}$ for the nonflat differential operators $\tilde{\nabla}^{2}$ and $\tilde{\Delta}_{L}$ are given in appendix B.

The convergence of the solutions is shown in figure 8. We again find smooth exponential convergence. Recall that the plotted quantities essentially give the error of the next lower resolution. Hence the next-to-highest resolution run with a total of $78^{3} \approx 500000$ collocation points has a maximum pointwise error of $\sim 0.5 \cdot 10^{-7}$. The wall clock time for that run is less than 2 hours on four RS 6000 processors.

This problem has also been attacked with a finite difference code[20]. The finite difference code required a runtime of $200 \mathrm{CPU}$ hours (on 16 nodes of an Origin 2000). The accuracy of the finite difference code seems to be comparable to the lowest resolution solve of our spectral solver, which took 16 minutes CPU time. Compared to the finite difference code the spectral code is almost embarrassingly fast. 


\section{IMPROVEMENTS}

The fact that spherical harmonics have fewer spectral coefficients than collocation points causes a host of complications. We have to solve for mixed real-spectral coefficients, Eq. (49). This complicates the operator $\mathcal{S}$, and severely complicates real space finite difference preconditioning. A double Fourier series 21 for the angular variables might be superior to the expansion in spherical harmonics, since this avoids the necessity for mixed real-spectral coefficients. Moreover one can then use fast transforms for both $\phi$ and $\theta$ which might improve runtime in the spherical shells.

We are working on cylinders as a third possible subdomain type. We also hope to use more general mappings that are no longer restricted to acting on each dimension separately.

In terms of pure runtime, one should try to optimize the interpolation to boundary points of overlapping subdomains. This is the part of the code that has the worst scaling with the number of unknowns. Replacing the explicit summation of the series by one of the faster methods discussed in [6] should speed up the code tremendously. As was seen in the discussion of parallelization in section 4.2.6, the code seems to be communication limited on a PC cluster. One should therefore also optimize the MPI calls. For example, one could overlap communication with subdomain internal computations.

Even without all these improvements our code is already fairly fast. This indicates the potential of our approach.

\section{CONCLUSION}

We have presented a new elliptic solver based on pseudo-spectral collocation. The solver uses domain decomposition with spherical shells and rectangular blocks, and can handle nonlinear coupled partial differential equations.

We introduced a new method to combine the differential operator, the boundary conditions and matching between subdomains in one operator $\mathcal{S}$. The equation $\mathcal{S} \underline{\mathbf{u}}=0$ is then solved with Newton-Raphson and an iterative linear solver. We show than one can employ standard software packages for nonlinear and linear solves and for preconditioning.

The operator $\mathcal{S}$ has the added benefit that it is modular. Therefore adaption of the method to a new PDE or to new boundary conditions is easy; the user has only to code the differential operator and/or the new boundary conditions. We also discuss our treatment of mappings which decouples mappings from the actual code evaluating the differential operator, and from the code dealing with basis functions and details of spectral transforms. This modularity again simplifies extension of the existing code with e.g. new mappings.

We demonstrated the capabilities of the new method with three examples on non-simply-connected computational domains in two and three dimensions and with one and four variables. We also demonstrated that the domain decomposition allows for vastly different length scales in the problem. During the examples we discussed various practical details like preconditioning and parallelization. Two of these examples were real applications from numerical relativity. We found the spectral code at the coarsest resolution to be as accurate as finite difference methods, but faster by one to two orders of magnitude. 


\section{APPENDIX A: PRECONDITIONING OF INVERSE MAPPINGS}

In a subdomain with inverse mapping that extends out to (almost) infinity, the outermost grid points are distributed very unevenly in physical space. This causes finite-difference approximations of derivatives to fail if they are based on the physical coordinate positions. Therefore we difference in the collocation coordinate $X$ and apply the mapping via Eq. (25). At the collocation grid point $X_{i}$ with grid spacing $h_{-}=X_{i}-X_{i-1}$ and $h_{+}=X_{i+1}-X_{i}$ we thus use

$$
\begin{aligned}
\left(\frac{\partial u}{\partial X}\right)_{i} & =-\frac{h_{+} u_{i-1}}{h_{-}\left(h_{-}+h_{+}\right)}+\frac{\left(h_{+}-h_{-}\right) u_{i}}{h_{+} h_{-}}+\frac{h_{-} u_{i+1}}{h_{+}\left(h_{-}+h_{+}\right)}, \\
\left(\frac{\partial^{2} u}{\partial X^{2}}\right)_{i} & =\frac{2 u_{i-1}}{h_{-}\left(h_{-}+h_{+}\right)}-\frac{2 u_{i}}{h_{-} h_{+}}+\frac{2 u_{i+1}}{h_{+}\left(h_{-}+h_{+}\right)}, \\
\left(\frac{\partial^{2} u}{\partial x^{2}}\right)_{i} & =X_{i}^{\prime 2}\left(\frac{\partial^{2} u}{\partial X^{2}}\right)_{i}+X_{i}^{\prime \prime}\left(\frac{\partial u}{\partial X}\right)_{i} .
\end{aligned}
$$

If one substitutes Eqs. (64) and (65) into (66), then the coefficients of $u_{i-1}, u_{i}$ and $u_{i+1}$ are the values that have to be entered into the FD-approximation matrix $\mathcal{A}_{F D}$.

Even with this trick, preconditioning of the radial derivatives in an extremely stretched outer sphere is not yet sufficiently good. The preconditioned Jacobian $\mathcal{B J}$ still contains eigenvalues of size $\sim 40$. The eigenmodes are associated with the highest radial mode in the outer sphere. We found that we can suppress these eigenvalues by damping this highest radial mode by a factor of 10 after the PETSc preconditioning is applied.

\section{APPENDIX B: PRECONDITIONING THE NONFLAT LAPLACIAN}

In a nonflat manifold, the Laplace operator of Eq. (59) contains second and first derivatives of $\psi$,

$$
\tilde{\nabla}^{2} \psi=\sum_{i, j=1}^{3} g^{i j} \frac{\partial^{2} \psi}{\partial x^{i} \partial x^{j}}+\sum_{i=1}^{3} f^{i} \frac{\partial \psi}{\partial x^{i}} .
$$

The coefficients $g^{i j}$ and $f^{i}$ are known functions of position. Since our particular manifold is almost flat, we have $g^{i i} \approx 1$, and $g^{i j} \approx 0$ for $i \neq j$. We base our preconditioning only on the diagonal part of (67),

$$
\sum_{i=1}^{3} g^{i i} \frac{\partial^{2}}{\partial x^{i^{2}}}
$$

In rectangular blocks, Eq. (68) can be preconditioned without additional fill-in in $\mathcal{A}_{F D}$. Inclusion of the mixed second derivatives from Eq. (67) in $\mathcal{A}_{F D}$ leads to a large fill-in of $\mathcal{A}_{F D}$. The increase in computation time due to the larger fill-in outweighs the improvement of convergence of the iterative solver in our problems.

For the spherical shells, matters are complicated by the fact that we use mixed real-space/spectral space coefficients [recall Eq. 449]]. It is easy to precondition the angular piece of the flat space Laplacian, since our basis functions $Y_{l m}$ are the eigenfunctions of this operator. Derivative operators with angle-dependent coefficients lead to convolutions in spectral space and lead thus to a large fill-in in the 
preconditioning matrix. Therefore we can only precondition radial derivatives with coefficients that are independent of the angles $\theta, \phi$. We thus need to approximate Eq. (68) by a flat space angular Laplacian and constant coefficient radial derivatives. We proceed as follows.

Rewrite Eq. (68) in spherical coordinates,

$$
\begin{aligned}
\sum_{i=1}^{3} g^{i i} \frac{\partial^{2}}{\partial x^{i^{2}}}= & G^{\theta \theta} \frac{\partial^{2}}{\partial \theta^{2}}+G^{\phi \phi} \frac{\partial^{2}}{\partial \phi^{2}}+G^{r r} \frac{\partial^{2}}{\partial r^{2}}+G^{\theta \phi} \frac{\partial^{2}}{\partial \theta \partial \phi} \\
& +G^{\theta r} \frac{\partial^{2}}{\partial \theta \partial r}+G^{\phi r} \frac{\partial^{2}}{\partial \phi \partial r}+F^{\theta} \frac{\partial}{\partial \theta}+F^{\phi} \frac{\partial}{\partial \phi}+F^{r} \frac{\partial}{\partial r} .
\end{aligned}
$$

At each grid point, the various functions $G$ and $F$ can be computed via standard Cartesian to polar coordinate transformations.

For each radial grid point $r_{i}$, average over the angles to obtain $\bar{G}_{i}^{\theta \theta}, \bar{G}_{i}^{r r}$ and $\bar{F}_{i}^{r}$. Now precondition as if $\bar{G}_{i}^{\theta \theta}$ were part of an angular piece of the flat space Laplacian, i.e. enter $-l(l+1) \bar{G}_{i}^{\theta \theta} / r_{i}^{2}$ as the diagonal element belonging to the unknown $\hat{u}_{i l m}$. Further, precondition $\bar{G}_{i}^{r r} \partial^{2} / \partial r^{2}+\bar{F}_{i}^{r} \partial / \partial r$ with finite differences as described in appendix A. Ignore all other terms in Eq. (69).

The operator $\tilde{\Delta}_{L}$ in Eq. (60) is defined by

$$
\tilde{\Delta}_{L} V^{i} \equiv \tilde{\nabla}^{2} V^{i}+\frac{1}{3} \sum_{k=1}^{3} \tilde{\nabla}^{i} \tilde{\nabla}_{k} V^{k}+\sum_{k=1}^{3} \tilde{R}_{k}^{i} V^{k},
$$

$\tilde{\nabla}$ and $\tilde{R}_{i j}$ being the covariant derivative operator and Ricci tensor associated with the metric of the manifold. $\tilde{\Delta}_{L} V^{i}$ contains thus the nonflat Laplace operator acting on each component $V^{i}$ separately, plus terms coupling the different components which involve second derivatives, too. We precondition only the Laplace operator $\tilde{\nabla}^{2} V^{i}$ for each component $V^{i}$ separately as described above and ignore the coupling terms between different components.

\section{ACKNOWLEDGMENTS}

We thank Gregory Cook for helpful discussions. This work was supported in part by NSF grants PHY-9800737 and PHY-9900672 to Cornell University. Computations were performed on the IBM SP2 of the Department of Physics, Wake Forest University, with support from an IBM SUR grant, as well as on the Platinum cluster of NCSA.

\section{REFERENCES}

[1] J. C. Adams and P. N. Swarztrauber. Spherepack 3.0. http://www.scd.ucar.edu/css/software/spherepack .

[2] O. Axelsson. Iterative solution methods. Cambridge University Press, 1994.

[3] S. A. Badeen. Kelp 1.3 home-page. http://wwwcse.ucsd.edu/groups/hpcl/scg/kelp.html .

[4] S. Balay, K. Buschelman, W. D. Gropp, D. Kaushik, L. C. McInnes, and B. F. Smith. PETSc home page. http://www.mcs.anl.gov/petsc , 2001. 
[5] M. Barrett, M. Berry, T. F. Chan, J. Van der Horst, and H. Van der Horst. Templates for the Solution of Linear Systems: Building Blocks for Iterative Methods. SIAM, Philadelphia, PA, 1994.

[6] J. B. Boyd. Chebyshev and Fourier Spectral Methods, 2nd edition. To be published by Dover, New York, 2001. For an online version, see http://wwwpersonal.engin.umich.edu/ jpboyd .

[7] S. Brandt and B. Brügmann. A simple construction of initial data for multiple black holes. Phys. Rev. Lett., 78(19):3606-3609, 1997.

[8] C. Canuto, M. Y. Hussaini, A. Quarteroni, and T. A. Zang. Spectral Methods in Fluid Dynamics. Springer, New York, 1987.

[9] G. B. Cook. Initial data for numerical relativity. Living Rev. Relativity, 3(5), 2000. [Online Article]: cited on Aug 11, 2001.

[10] G. B. Cook, M. W. Choptuik, M. R. Dubal, S. Klasky, R. A. Matzner, and S. R. Oliveira. Three-dimensional initial data for the collision of two black holes. Phys. Rev. D, 47(4):1471-1490, 1993.

[11] P. Demaret and M. O. Deville. Chebyshev collocation solutions of the NavierStokes equations using multi-domain decomposition and finite element preconditioning. J. Comp. Phys, 95:359-386, 1991.

[12] M. Deville and E. Mund. Chebyshev pseudospectral solution of second-order elliptic equations with finite element preconditioning. J. Comp. Phys, 60:517$533,1985$.

[13] D. Funaro, A. Quarteroni, and P. Zanolli. An iterative procedure with interface relaxation for domain decomposition methods. SIAM J. Numer. Anal., 25(6):1213-1236, Dec. 1988.

[14] P. Gervasio, E. Ovtchinnikov, and A. Quarteroni. The spectral projection decomposition method for elliptic equations in two dimensions. SIAM J. Numer. Anal., 34(4):1616-1639, 1997.

[15] D. Gottlieb and S. A. Orszag. Numerical Analysis of Spectral Methods:Theory and Applications. Society of Industrial and Applied Mathematics, 1977.

[16] P. Grandclément, S. Bonazzola, E. Gourgoulhon, and J.-A. Marck. A multidomain spectral method for scalar and vectorial poisson equations with noncompact sources. J. Comp. Phys, 170:231-260, 2001.

[17] L. E. Kidder and L. S. Finn. Spectral methods for numerical relativity: The initial data problem. Phys. Rev. D, 62(8):084026, 2000.

[18] H.-C. Ku. Solution of flow in complex geometries by the pseudospectral element method. J. Comp. Phys, 117:215-227, 1995.

[19] M. G. Macaraeg and C. L. Streett. Improvements in spectral collocation discretization through a multiple domain technique. Appl. Numer. Math, 2:95$108,1986$.

[20] P. Marronetti and R. A. Matzner. Solving the initial value problem of two black holes. Phys. Rev. Lett., 85(26):5500-3, 2000. 
[21] S. Orszag. Fourier series on spheres. Monthly Weather Review, 102(1):56-75, 1974.

[22] S. A. Orszag. Spectral methods for problems in complex geometries. J. Comp. Phys, 37:70, 1980.

[23] H. P. Pfeiffer, S. Teukolsky, and G. B. Cook. Comparing initial data sets for binary black holes. In preparation.

[24] A. Pinelli, A. Vacca, and A. Quarteroni. A spectral multidomain method for the numerical simulation of turbulent flows. J. Comp. Phys, 136:546-558, 1997.

[25] W. H. Press, S. A. Teukolsky, W. T. Vetterling, and B. P. Flannery. Numerical Recipes in C. Cambridge University Press, Cambridge, UK, 1997.

[26] Y. Saad. A flexible inner-outer preconditioned GMRES algorithm. SIAM J. Sci. Comput, 14(2):461-469, 1993.

[27] B. F. Smith, P. Bjørstad, and W. Gropp. Domain Decomposition: Parallel Multilevel Methods for Elliptic Partial Differential Equations. Cambridge University Press, 1996.

[28] P. N. Swarztrauber. On the spectral approximation of discrete scalar and vector functions on the sphere. SIAM J. Numer. Anal., 16(6):934-949, 1979.

[29] P. N. Swarztrauber. The approximation of vector functions and their derivatives on the sphere. SIAM J. Numer. Anal., 18(2):191-210, 1981. 